\title{
Saturated Fatty Acid Inhibits Viral Replication in Chronic Hepatitis B Virus Infection With Nonalcoholic Fatty Liver Disease by Toll-Like Receptor 4-Mediated Innate Immune Response
}

\author{
Rui-Nan Zhang ${ }^{1}$; Qin Pan ${ }^{1}$; Zheng Zhang ${ }^{1}$; Hai-Xia Cao ${ }^{1}$; Feng Shen ${ }^{1}$; Jian-Gao Fan ${ }^{1, *}$ \\ ${ }^{1}$ Shanghai Key Laboratory of Children's Digestion and Nutrition, Department of Gastroenterology, XinHua Hospital, Shanghai Jiao Tong University School of Medicine, Shanghai, \\ China \\ *Corresponding Author: Jian-Gao Fan, Shanghai Key Laboratory of Children's Digestion and Nutrition, Department of Gastroenterology, XinHua Hospital, Shanghai Jiao Tong \\ University School of Medicine, Shanghai, China. Tel/Fax: +86-2125077340, E-mail: fattyliver2004@126.com
}

Received: February 12, 2015; Revised: April 9, 2015; Accepted: April 17, 2015

\begin{abstract}
Background: Chronic Hepatitis B (CHB) infection is common in patients with Non-Alcoholic Fatty Liver Disease (NAFLD). The replication level of Hepatitis B Virus (HBV) was inversely correlated with hepatic steatosis. Toll-Like Receptor (TLR) 4-mediated innate immunity plays a pivotal role in the occurrence of NAFLD and controls HBV replication.

Objectives: This study aimed to investigate whether the TLR4-mediated innate immunity stimulates the pathogenesis of CHB in patients with NAFLD and to determine whether TLR4 plays a role in inhibiting HBV replication.

Materials and Methods: The HBV transgenic mice were randomized into the HBV and HBV/NAFLD groups. HepG2.2.15 cells were treated with different concentrations $(0-200 \mu \mathrm{M})$ of Stearic Acid (SA) to induce steatosis. The total RNA of the liver tissue was extracted for RealTime Polymerase Chain Reaction (RT-PCR) detection, and immunohistochemistry or western blot was conducted for further validation. The Enzyme-Linked Immunosorbent Assay (ELISA) analysis was applied to evaluate the production of Interleukin 6 (IL-6), Tumor necrosis factor $\alpha$ (TNF- $\alpha$ ) and Interferon $\beta$ (IFN- $\beta$ ). Moreover, viral dynamics were analyzed using HBV DNA and HBV-related antigens (HBsAg and HBeAg).

Results: Non-alcoholic fatty liver disease was induced in HBV-transgenic mice fed with High Fat Diet(HFD) for 8-24 weeks. Oil red-O staining positive droplets and the content of Triglyceride (TG) were increased in HepG2.2.15 cells treated with SA. TLR4, Myeloid differentiation factor 88 (MyD88), IL-6 and TNF- $\alpha$ expression levels were significantly higher in the HBV/NAFLD group and the steatotic HepG2.2.15 cells than those in their respective controls. Compared to the HBV group, significant reductions in serum levels of HBsAg, HBeAg, and HBV DNA titers occurred in the HBV/NAFLD group at 24 weeks, but the IFN- $\beta$ level was remarkably increased. Similar data were also obtained from the steatoric HepG2.2.15 cells.

Conclusions: Saturated Fatty Acids (SFAs) served as a potential ligand for TLR4 and activated TLR4 signaling pathway, which might be involved in the pathogenesis. Thus, SFAs can accelerate the mechanism of inhibiting HBV replication in CHB with NAFLD.
\end{abstract}

Keywords: Diet, High-Fat; Stearic Acid; Toll-like receptor 4; Hepatitis B Virus; Nonalcoholic Fatty Liver Disease

\section{Background}

Chronic Hepatitis B (CHB) is a global health concern as it is associated with substantial morbidity and mortality. Although viral factors, such as Hepatitis B Virus (HBV) DNA, HBeAg, and genotype increase the risk of histological progression and Hepatocellular Carcinoma (HCC), there are other host factors that can also play key roles in the development of HCC (1-4). Non-Alcoholic Fatty Liver Disease (NAFLD) is increasingly common in both the general population and the HBV-infected patients, reflecting the increasing prevalence of obesity in both Western and Eastern countries $(5,6)$. The spectrum of NAFLD ranges from simple hepatic steatosis through Non-Alcoholic Steato Hepatitis (NASH) to liver cirrhosis $(1-3,7)$. Nowadays, many Asian patients are suffering from both NAFLD and chronic HBV infection. However, the mechanism of NAFLD and its effects on HBV infection have not yet been adequately clarified in patients with CHB. Some previ- ous reports have revealed that hepatic steatosis in $\mathrm{CHB}$ patients is mainly associated with metabolic disorders, such as obesity, Type 2 Diabetes Mellitus (T2DM), and dyslipidemia; it is not associated with viral load or genotype. This is proved by the strongly expressive results indicating a negative association of hepatic steatosis with viral load. Thus, hepatic steatosis may enhance viral clearance and inhibit HBV DNA replication $(1,5,7,8)$. However, we have not been successful in elucidating the mechanism underlying the association between steatosis and HBV.

Some evidences indicate that Toll-Like Receptor 4 (TLR4) signals the pathway associated with the pathogenesis of NAFLD in patients with HBV infection. TLRs are a family of pattern recognition receptors that play a critical role in the innate immune system as ten different types of TLRs are expressed in human beings. Toll-like receptor 4 is a cell surface receptor that is crucial for the activation of

Copyright ( ) 2015, Kowsar Corp. This is an open-access article distributed under the terms of the Creative Commons Attribution-NonCommercial 4.0 International License (http://creativecommons.org/licenses/by-nc/4.0/) which permits copy and redistribute the material just in noncommercial usages, provided the original work is properly cited. 
innate immune responses (9). This receptor binding sites activate two distinct intracellular signaling pathways via MyD88-dependent and MyD88-independent pathways, resulting in the induction of proinflammatory cytokine genes, such as Tumor Necrosis Factor $\alpha$ (TNF- $\alpha$ ), Interleukin 6 (IL-6), and Type 1 Interferons (IFNs I) $(10,11)$. Bacterial endotoxin Lipopolysaccharide (LPS) is a well-known TLR4 ligand $(12,13)$. Nonbacterial substances such as Free Fatty Acids (FFAs) may also function as TLR4 ligands. Several studies have reported that Palmitic Acid(PA) and Oleic Acid (OA) are the most common FFAs that covalently coordinate with TLR4 to induce the expression of proinflammatory cytokines in macrophages, adipocytes, and liver cells $(9,14-16)$. It has been reported that TLR4/MyD88 signaling pathways played a pivotal role in the development of NAFLD. Furthermore, downstream proinflammatory cytokines (TNF $\alpha$, IL-6) promoted the progression of NAFLD. However, the interaction between chronic HBV infection and TLR4 is complex; some studies have reported that HBV replication is inhibited when LPS signals TLR4 to up-regulate IFN- $\beta$ expression levels through MyD88independent pathway (17-19).

It has been reported in previous studies that both direct and indirect mediators inhibit viral replication. IFN $\alpha / \beta$, TNF $\alpha$, IL-1, and nitric oxide act as direct mediators, whereas immunoregulatory cytokines, chemokines, lipid mediators, and peroxide act as indirect antiviral mediators $(19,20)$. We speculated that IFN- $\beta$, IL-6, and TNF- $\alpha$ served as the mediators to inhibit HBV replication in patients having chronic HBV infection along with NAFLD. In NAFLD patients and animal models, increased circulating LPS and FFAs, which are recognized by TLR4, lead to the elevated levels of IFN- $\beta$, TNF- $\alpha$ and IL-6. Therefore, we also speculated that both TLR4/MyD88 dependent and independent pathways might be simultaneously activated in chronic HBV infection with NAFLD.

\section{Objectives}

In this study, our aim was to determine whether TLR4 signaling pathway participated in the occurrence of NAFLD in HBV transgenic mice; we also identified whether Stearic Acid (SA) acts as a ligand of TLR4 to induce the steatosis of HepG2.2.15 cells. In addition, we determined whether TLR4-mediated innate immunity inhibited HBV replication in chronic HBV infection with NAFLD.

\section{Materials and Methods}

\subsection{Mice and Experimental Procedures}

We purchased male HBV-transgenic Balb/C mice aged 4-weeks from the Transgenic Engineering Research Laboratory, $458^{\text {th }}$ Hospital of PLA (Guangzhou, China) and divided them into the following two groups, that is, control group and NAFLD group for 8-24 weeks of treatment. The mice belonging to the control group (HBV group, $n$ $=8$ ) were fed standard chow. The mice belonging to the
NAFLD group (HBV/NAFLD group, $\mathrm{n}=8$ ) were fed High Fat Diet (HFD). This HFD consisted of $2 \%$ cholesterol, $10 \%$ lard oil, and $88 \%$ standard chow by weight. All the animals received unlimited access to the chow and water. These mice were sacrificed at the end of 8,16 , and 24 weeks of the study, respectively. We collected their serum samples and stored them at $-20^{\circ} \mathrm{C}$ for further use. A portion of the liver's left lobe was snapped and frozen in liquid nitrogen for performing subsequent RNA or protein analyses, and the remaining portion of this lobe was used to prepare homogenates. All the experiments were performed in accordance with the procedure approved by the Shanghai Jiao Tong University Institutional Animal Care and Use Committee.

\subsection{Cell Culture}

HepG2.2.15 cells were obtained from the American Type Culture Collection (Manassas, VA). These cells were maintained in a humidified incubator containing an atmosphere of 5\% $\mathrm{CO}_{2}$ and a Dulbecco's modified Eagle's medium (DMEM) supplemented with L-glutamine, penicillin-streptomycin, and 10\% fetal bovine serum (FBS, Gibco BRL, USA); the temperature of this medium was maintained at $37^{\circ} \mathrm{C}$.

\subsection{Cell Proliferation Assay}

Cell proliferation was assessed using 3 - [4,5-dimethylthiazol-2-yl] - 2, 5-diphenyl tetrazolium bromide (MTT; Sigma, USA). HepG2.2.15 cells were seeded onto a 96-well plate at $4 \times 10^{3} /$ well in triplicate wells. After 24 hours, the cells were incubated with $25 \mu \mathrm{M}, 50 \mu \mathrm{M}, 100 \mu \mathrm{M}$, and 200 $\mu \mathrm{M}$ of SA (Sigma, USA), which was then dissolved in Dimethyl Sulfoxide (DMSO); the reaction mixture was kept standing for 6 hours, 24 hours, or 48 hours. Thereafter, 5 $\mathrm{mg} / \mathrm{mL}$ of MTT was added to the culture medium according to method recommended by the manufacturer, and the cells were then incubated for an additional 4 hours. At the end of the assay, the cell-growth medium was replaced with $150 \mu \mathrm{L}$ DMSO and the absorbance values were recorded at $490 \mathrm{~nm}$.

\subsection{Oil Red-O Staining and Triglyceride Assay}

After HepG2.2.15 cells were treated with SA $(0 \mu \mathrm{M}, 25 \mu \mathrm{M}$, $50 \mu \mathrm{M}, 100 \mu \mathrm{M}$, and $200 \mu \mathrm{M})$ for 48 hours, the degree of steatosis of cells was measured using Oil red-O staining. In this assay, cells were fixed with formaldehyde, while lipids were stained using 0.5\% Oil red-O in isopropyl alcohol for 20 minutes, and nuclei were counterstained with hematoxylin for 1 minute. Cell images were then observed under a bright field microscope (Olympus, Japan) at $200 \times$ magnification. Total triglyceride (TG) levels were measured by performing an enzymatic assay using a TG assay kit from Applygen Technologies Inc. (Shanghai, China), according to the manufacturer's instructions, and the TG concentrations were normalized with protein content. 


\subsection{Histopathological and Immunohistochemistry Analysis}

Ten percent formalin fixed, paraffin embedded sections of hepatic tissue were stained with hematoxylin-eosin ( $\mathrm{H}$ and $\mathrm{E}$ ) using standard procedures. The HBV/NAFLD groups were blindly evaluated using the NAFLD activity score.

Paraffin-embedded liver tissues were deparaffinized with xylene, washed with graded ethanol and then permeabilized with $0.2 \%$ trypsin at room temperature for 30 minutes. Inactivation of endogenous peroxidase was achieved using $3 \% \mathrm{H}_{2} \mathrm{O}_{2}$ for 20 minutes. Then, these tissue sections were washed with Phosphate-Buffered Saline (PBS) and incubated overnight with appropriate primary antibodies at $4^{\circ} \mathrm{C}$. Goat antibodies raised against mouse TLR4 or IL-6 (Abcam, Cambridge, UK), or mouse MyD88 (Cell signaling, USA) were diluted 1:100 in PBS. While using secondary antibodies, PBS served as the negative control. 3, 3-di-amino-benzidine (DAB; Santa Cruz, USA) was used for visualization. All the sections were evaluated using a binocular microscope (Leica, Germany). We quantitatively analyzed the intensity of immunostaining for TLR4, MyD88, and IL-6 using images of the liver tissues obtained from the two groups of mice at 8,16, and 24 weeks, respectively. These images were analyzed for positive staining at a magnification of $200 \times$ using the quantitative immunohistochemical analysis software Image Pro Plus (Media Cybernetics, Baltimore, MD). We evaluated integrated optical densities, where the term "integrated" refers to the sum of all pixel intensities or density values in a given region.

\subsection{Quantitative Real-Time Polymerase Chain Re- action}

Total RNA was extracted from the liver tissues and HepG2.2.15 cells using the TRIzol reagent (Invitrogen, USA) and reverse transcribed using the PrimeScript RT Reagent Kit (TaKaRa, Kusatsu, Japan). The mRNA expression of TLR4, MyD88, IL-6, TNF- $\alpha$, and IFN- $\beta$ was measured by Real-Time Polymerase Chain Reaction (RT-PCR) using the SYBR Premix Ex TaqTM Kit (TaKaRa, Kusatsu, Japan) and an ABI 7500 RT-PCR System (Applied Biosystems, USA). Target mRNA levels were normalized to $\beta$-actin expression levels. A duplicate of this experiment was performed for the same reaction. The $2^{-\Delta \Delta \mathrm{Ct}}$ method was used to calculate the relative expression levels for each gene. The sequences of primers were available upon request.

\subsection{Enzyme-Linked Immunosorbent Assay}

The Enzyme-Linked Immunosorbent Assay (ELISA) DuoSet Kits (R and D Systems Inc., Minneapolis, MN, USA) were used to determine the concentrations of TNF- $\alpha$ and IL-6 in hepatic homogenates and that of the cell culture supernatants and IFN- $\beta$ in HBV transgenic mice serum and cell supernatants. The process of measuring these concentrations was performed according to the manufacturer's instructions. All experiments were performed in duplicate.

\subsection{Western Blot Analysis}

The harvested cells were lysed in radioimmunoprecipitation (RIPA) buffer containing the protease inhibitor PMSF. Protein concentrations were determined using 2-Quinolinecarboxylic acid (BCA) assays, and samples containing $40 \mu \mathrm{g}$ of total protein were resolved using $10 \%$ sodium dodecyl sulfate polyacrylamide gradient gel (SDS-PAGE); these resolved samples were then transferred to nitrocellulose membranes. The nitrocellulose membranes were blocked for 2 hours and incubated with 1:1000 rabbit polyclonal antihuman TLR4 (Abcam, Cambridge, UK), MyD88 (Cell signaling, USA), and mouse monoclonal antihuman $\beta$-actin (Beyotime, Shanghai, China) overnight at $4^{\circ} \mathrm{C}$. Thereafter, these membranes were washed and incubated at room temperature with a Horseradish Peroxidase (HRP)-conjugated anti-goat secondary antibody (Beyotime, Shanghai, China) for 90 minutes. The detection was performed using enhanced chemiluminescence (ECL) detection reagents and a ChemiDoc Imaging System (Bio-Rad Laboratories, Hercules, CA).

\subsection{Hepatitis B Virus-DNA Titers Assay}

Serum of HBV transgenic mice and cell supernatants of HepG2.2.15 were corrected; then lysis buffer was added into the HBV-DNA Detection Kit (Daangene, Guangzhou, China). The HBV-DNA levels were measured by RT-PCR, according to the manufacturer's instructions. The threshold cycle values were used to determinate the concentration of HBV-DNA. This experiment was performed in triplicate.

\subsection{Statistical Analysis}

The data were expressed in terms of Mean \pm SD. An unpaired t-test was performed to compare the expression levels of mRNA and protein with the respective levels observed in the control groups. The analyses were performed using GraphPad Prism 5 software (GraphPad Software, Inc. San Diego, CA, USA). The values of $\mathrm{P}<0.05$ were considered to be statistically significant.

\section{Results}

\subsection{Establishment of Non-Alcoholic Fatty Liver Dis- ease in Hepatitis B Virus Transgenic Mice}

Compared with the HBV transgenic mice in the control group, the mice belonging to the HBV/NAFLD group gained weight gradually and experienced an elevation in serum aminotransferases, dyslipidemia, impaired fasting glucose, and abnormal liver histology within 8 
to 24 weeks of the treatment. In the HBV/NAFLD group, the mice experienced significant macrovesicular and microvesicular steatosis within 8 weeks; they developed steatohepatitis in 16 weeks and steatohepatitis with pericellular fibrosis in 24 weeks. However, the control mice fed with standard chow showed normal liver histology during the treatment period that extended from 8 to 24 weeks. The results of serum biochemistry and hepatic histopathological analyses have been reported in our previous study (4).

\subsection{Up-Regulated Expressions of Toll-like receptor 4} (TLR4), Myeloid differentiation factor 88 (MyD88), Interleukin 6 (IL-6) and Interferon- $\beta$ (IFN- $\beta$ ) in Hepatitis B Virus/Nonalcoholic Fatty Liver Disease (HBV/NAFLD) Groups

As compared to that of HBV group, the HBV/NAFLD group demonstrated significantly higher expression of TLR4, MyD88, and IL-6 in the tissue samples analyzed for mRNA and protein levels at the end of 8,16 , and 24 weeks $(\mathrm{P}<0.05)$ (Figures 1 and 2). The quantitative data for TLR4, MyD88, and IL-6 obtained by immunohistochemical staining we analyzed using Image Pro Plus 6.0. The Integrated Optical Density (IOD) values further confirmed the increased levels of TLR4, MyD88, and IL-6 in liver tissues, respectively, with the IOD values exemplifying a time- dependent manner $(\mathrm{P}<0.05)$ (Figure 2). Moreover, the expressions of TLR4, MyD88, and IL-6 in the HBV/NAFLD group experienced obvious up-regulation during the HFD treatment (Figures 1 and 2). The mRNA and protein expressions of TNF- $\alpha$ in the HBV/NAFLD group also exhibited an increasing trend, though no significant, when compared with that in the HBV group at different timepoints (Figure 1). Then, the significantly elevated levels of serum IFN- $\beta$ were also detected at week 24 (Figure 1G).

4.3. Up-Regulation of Toll-like receptor 4 (TLR4), Myeloid differentiation factor 88 (MyD88), Interleukin 6 (IL-6) and Tumor necrosis factor $\alpha$ (TNF- $\alpha$ ) in Steatotic HepG2.2.15 Cells

The biological effects of SA $(25-200 \mu \mathrm{M})$ were first analyzed using the cell proliferation assays. The data showed that SA $(25-200 \mu \mathrm{M})$ promoted the HepG2.2.15 cell proliferation in a time-dependent manner. Furthermore, at different time points, the cell growth rate was relatively lower at $200 \mu \mathrm{M}$ SA than at any other concentrations (Figure $3 \mathrm{~B}$ ). Therefore, we adopted the time points of 48 hours for further experiments. After being exposed to 25 - $200 \mu \mathrm{M}$ SA for 48 hours, both the number of Oil red-O staining positive droplets and the content of Triglyceride (TG) in cell lysates were significantly increased in HepG2.2.15 cells in a dose-dependent manner, compared to the control cells (Figure $3 \mathrm{~A}$ ).

Figure 1. The Expression of Toll-like receptor 4 (TLR4), Myeloid differentiation Factor 88 (MyD88), Interleukin 6 (IL-6) and Interferon- $\beta$ (IFN- $\beta$ ) in the Hepatitis B Virus/Nonalcoholic Fatty Liver Disease (HBV/NAFLD) Groups
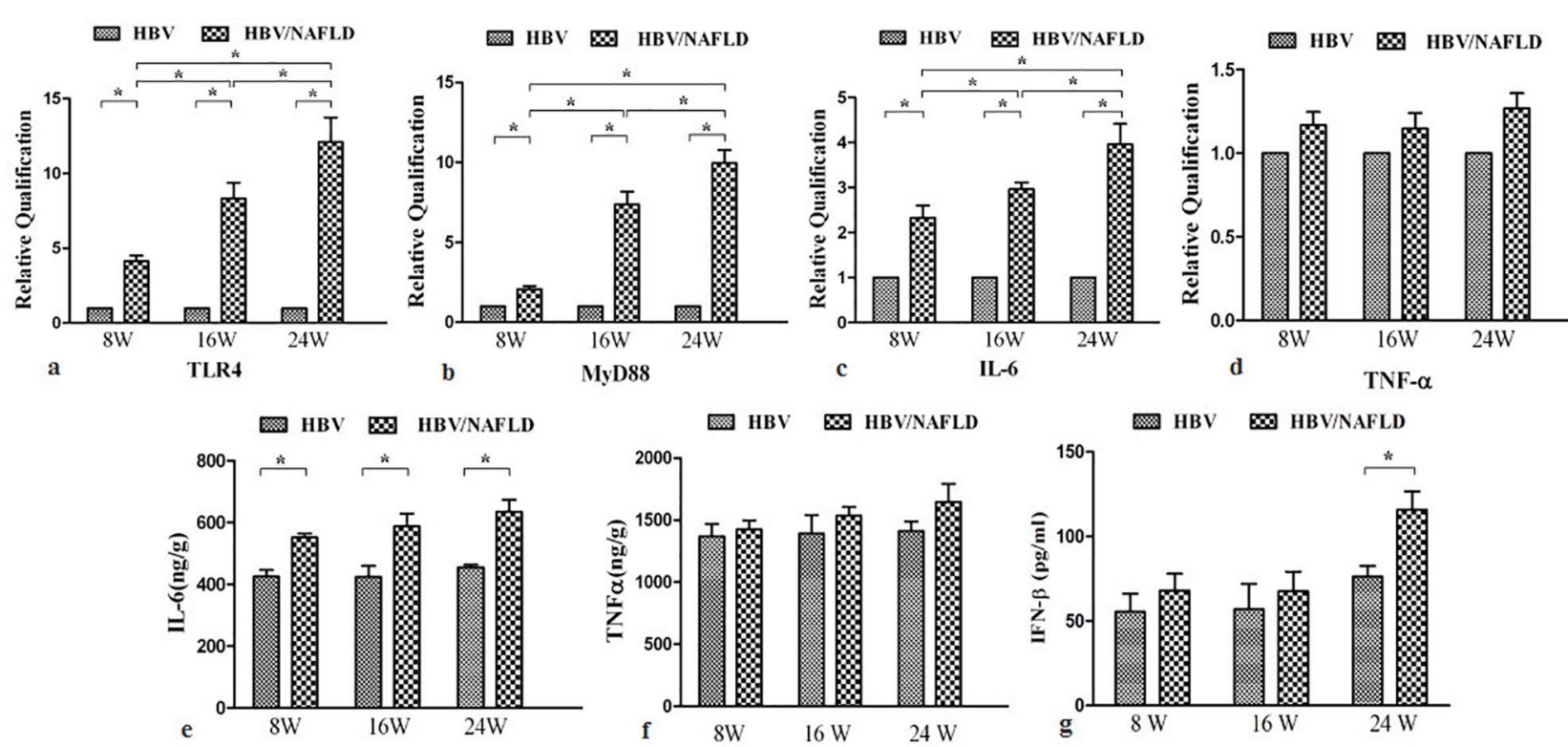

A, B, C and D, RT-PCR showed mRNA expression changes of TLR4, MyD88, IL-6, and TNF- $\alpha$ in the livers of the HBV and HBV/NAFLD groups at 8, 16 and 24 weeks. E, and F, The concentration of pro-inflammatory cytokines (pg/g liver tissue) were measured by ELISA in liver homogenates of HBV and HBV/NAFLD groups at 8,16 , and 24 weeks. The data are expressed in terms of Mean \pm Standard Error $(\mathrm{SE})(\mathrm{n}=8)$, and all are normalized to HBV groups at different time-points. ${ }^{*} \mathrm{P}<0.05$. 
Figure 2. Hepatic Immunohistochemical Staining for Toll-like receptor 4 (TLR4), Myeloid differentiation factor 88 (MyD88) and Interleukin 6 (IL-6) in Hepatitis B Virus/Nonalcoholic Fatty Liver Disease (HBV/NAFLD) Groups
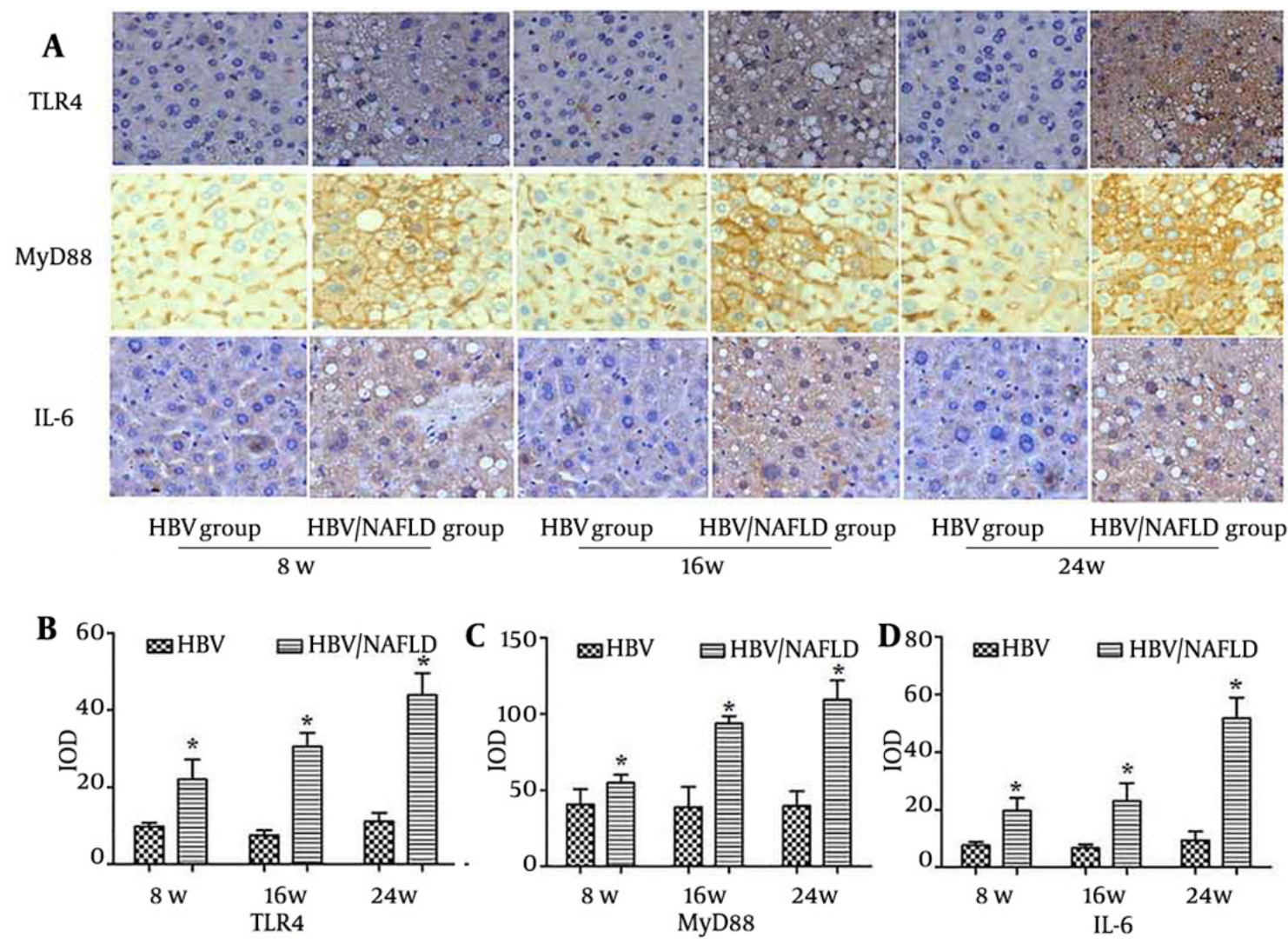

A, the expression of TLR4, MyD88, and IL-6 in the livers of the HBV and HBV/NAFLD groups at 8, 16, and 24 weeks. Representative histological sections of immunohistochemistry (IHC) staining of livers were shown. B, C and D, quantitative data for TLR4, MyD88, and IL-6 immunohistochemical staining. Integrated optical densities (IOD) values of TLR4, MyD88, and IL-6 expression were determined using Image Pro Plus 6.0. Data are expressed as the Mean \pm Standard Error $(\mathrm{SE})(\mathrm{n}=8)$, and all are normalized to HBV groups at different time-points. ${ }^{*} \mathrm{P}<0.05$.

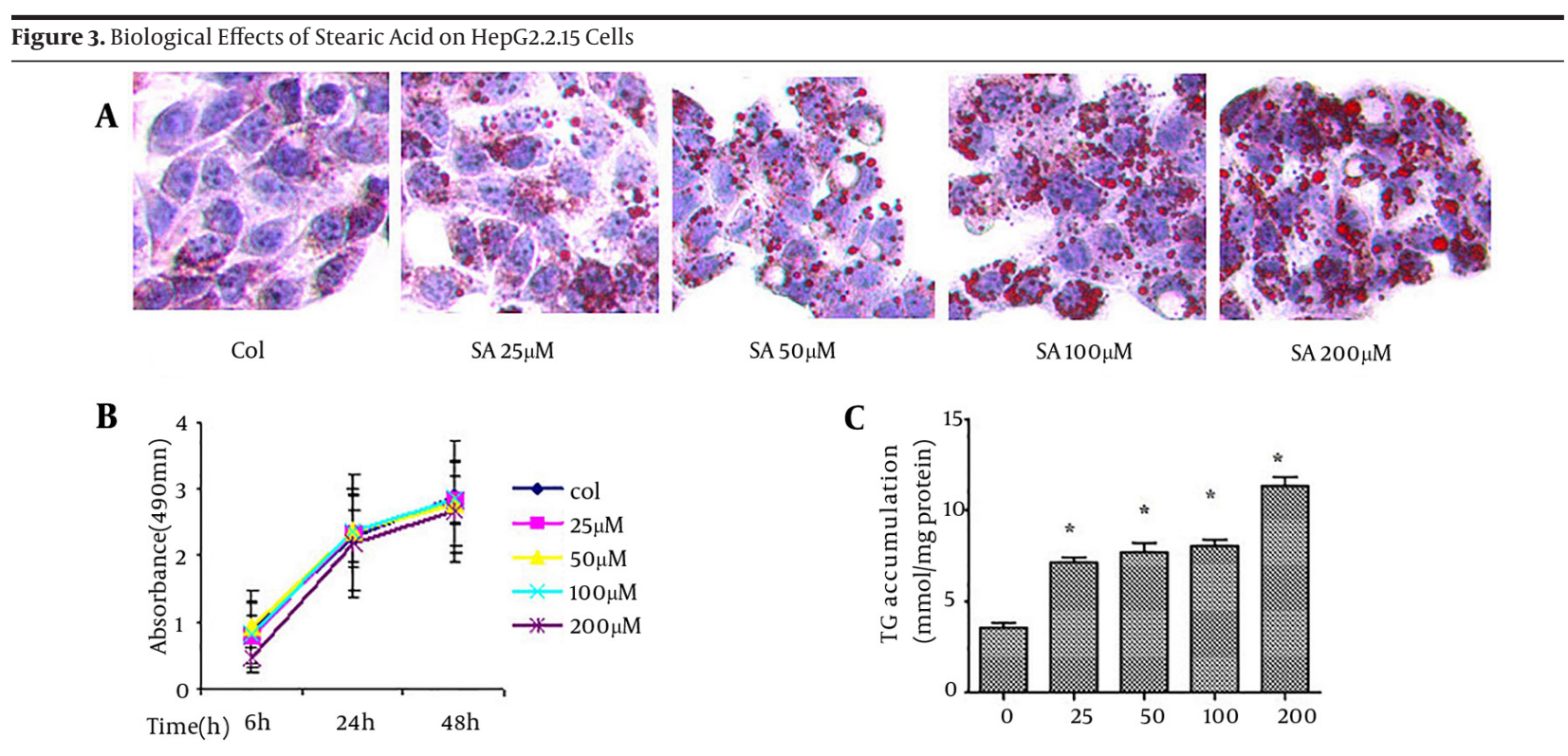

A, Representative images show the lipid accumulation in HepG2.2.15 cells following SA treatment, as revealed by Oil Red O staining; B, Cell proliferation assay in HepG2.2.15 cells with/without SA incubation at 6 hours, 24 hours, and 48 hours; C, TG content (mmol/mg protein) measurement in HepG2.2.15 cells; data are expressed as the Mean \pm Standard Error (SE) in triplicate. ${ }^{*} \mathrm{P}<0.05$. 
Compared to the control cells, the mRNA and protein levels of TLR4 and MyD88 were higher in steatotic HepG2.2.15 cells at different concentrations of SA after 48 hour (Figure 4). The expression of downstream proinflammatory cytokines IL- 6 and TNF- $\alpha$ also significantly increased in steatotic HepG2.2.15 cells (Figure 4). Then, the IFN- $\beta$ expression levels were also detected in steatotic HepG2.2.15 cells, and cell supernatants showed that the level elevated remarkably especially at $50-200 \mu \mathrm{M}$ concentrations of SA (Figure 4).

\subsection{Viral Characterization in Hepatitis B Virus Transgenic Mice With Nonalcoholic Fatty Liver Disease and HepG2.2.15 Cells With Stearic Acid- Induced Steatosis}

When compared to the HBV group, the viral indices
(HBsAg, HBeAg and HBV-DNA) of the HBV/NAFLD group showed a decreasing tendency during the time-period of 8 - 16 weeks. Whereas significant decrease in the viral indices occurred at the end of the 24 weeks (Figure 5). However, there were no significant differences of HBsAg and HBeAg in the liver tissue that was obtained at different stages from the HBV and the HBV/NAFLD groups. The viral indices except HBsAg had no difference when compared at 16 weeks and 24 weeks to 8 weeks.

After incubating HepG2.2.15 cells with $25 \mu \mathrm{M}, 50 \mu \mathrm{M}$, $100 \mu \mathrm{M}$, and $200 \mu \mathrm{M}$ SA for 48 hours, HBsAg, HBeAg, and HBV-DNA titers were determined. The results indicated that the viral dynamic exhibited a tendency of reduction in steatotic HepG2.2.15 cells, reducing significantly at $100 \mu \mathrm{M}$ and $200 \mu \mathrm{M}$ SA. This reduction in viral dynamics was not observed in HepG2.2.15 cells without SA (Figure 5).

Figure 4. The mRNA and Protein Expression Levels of Toll-like receptor 4 (TLR4), Myeloid differentiation factor 88 (MyD88), Interleukin 6 (IL-6), Tumor necrosis factor $\alpha$ (TNF- $\alpha)$ and Interferon- $\beta$ (IFN- $\beta$ ) in Steatotic HepG2.2.15 Cells

A

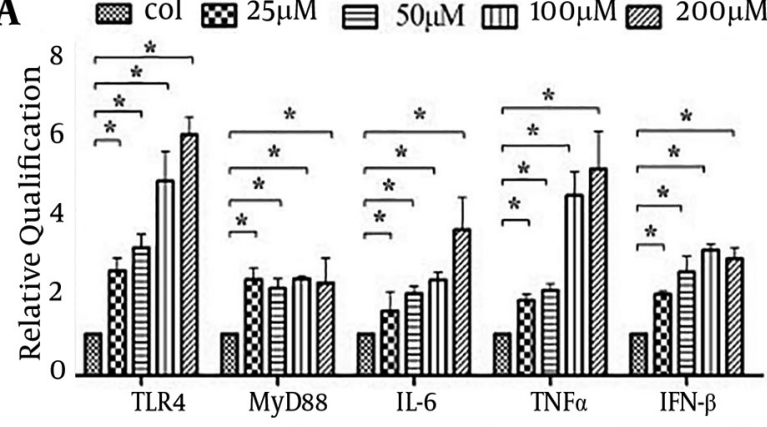

C

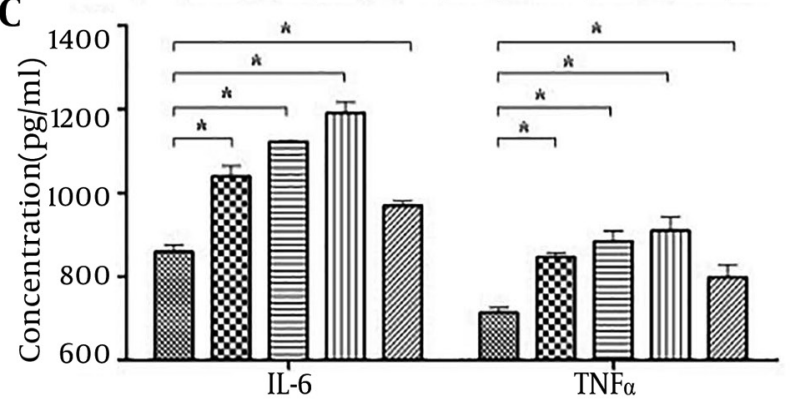

B

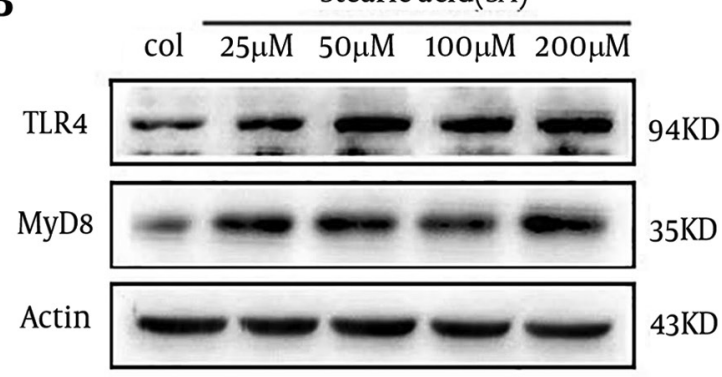

A, RT-PCR showed mRNA expression changes of TLR4, MyD88, IL-6, TNF- $\alpha$, and IFN- $\beta$ in HepG2.2.15 cells with/without SA incubation for 48 hours; B, The expression of TLR4 and MyD88 proteins was analyzed by western blot in HepG2.2.15 cells with/without SA incubation for 48 hours; Also, C, D, The concentration of proinflammatory cytokines and IFN- $\beta(\mathrm{pg} / \mathrm{mL})$ were measured by ELISA in cell culture supernatants of HepG2.2.15 cells with/without SA incubation for 48 hours. Data are expressed as the Mean \pm Standard Error (SE) in triplicate. ${ }^{*} \mathrm{P}<0.05$. 

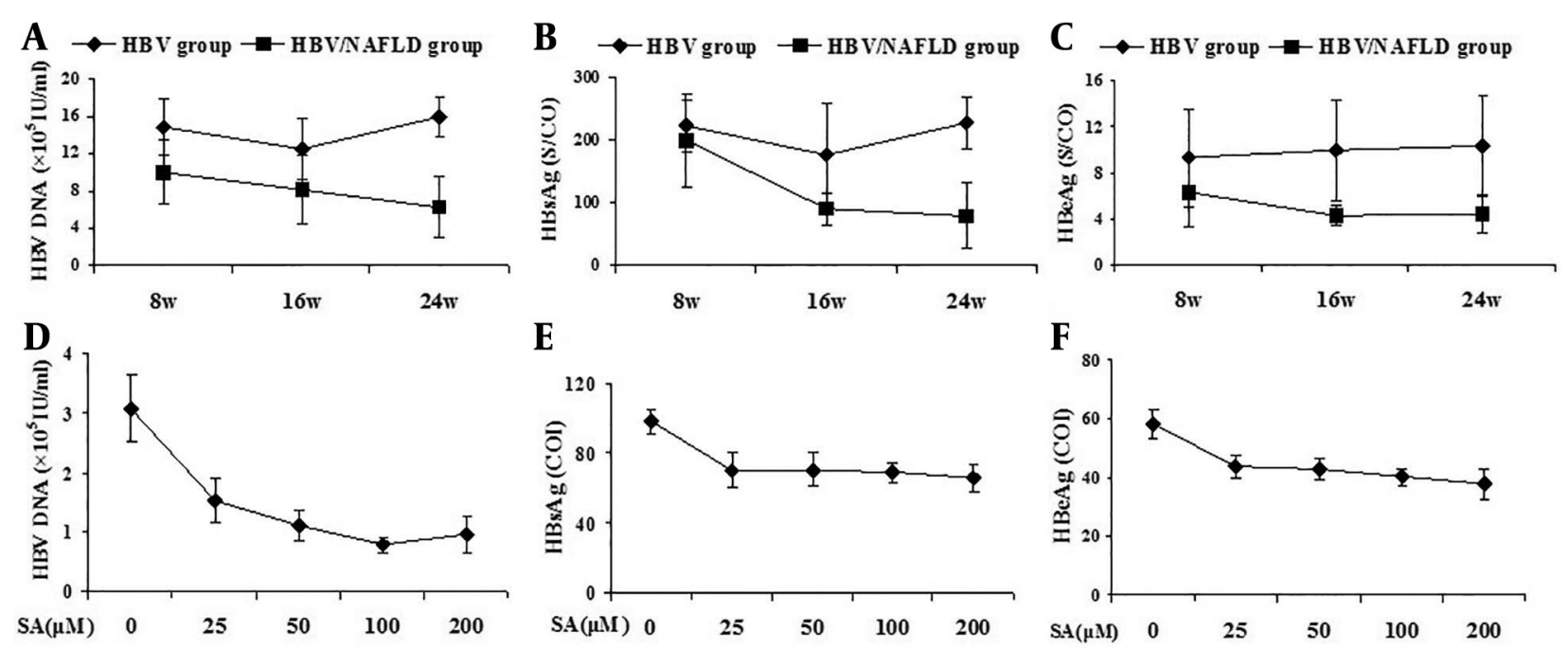

A, B, C, effects of HFD on the serum viral dynamics (HBV DNA, HBsAg and HBeAg) of HBV transgenic mice; C, D, F, Effects of different concentrations of SA $(25 \mu \mathrm{M}, 50 \mu \mathrm{M}, 100 \mu \mathrm{M}$, and $200 \mu \mathrm{M})$ on the supernatant viral dynamics of HepG2.2.15 cells. ${ }^{*} \mathrm{P}<0.05$.

\section{Discussion}

The pathogenesis of NAFLD in patients with chronic HBV infection is not well-understood, and for completely ethical and practical reasons, it has been difficult to conduct detailed mechanistic studies in humans. Therefore, great attempts have been made to develop in vivo and in vitro models of HBV infection with NAFLD in order to explore their pathogenesis and interaction. Some models have shown encouraging results. In this study, the HBV transgenic mice were immune-tolerant to HBV-related antigens as they had normal innate and acquired immunity and the liver histology of these mice was also normal $(5,21)$. HepG2.2.15 is an immortalized hepatocyte line established by the stable transfection of HBV ayw strain genome in HepG2 cells. In this study, we developed a model of NAFLD in HBV transgenic mice by continuously HFD. Furthermore, an in vitro steatosis developed in HepG2.2.15 cells treated with SA. These models closely resembled the occurrence of NAFLD in patients suffering from persistent chronic HBV infection.

There have been several evidences to indicate that TLRs plays a critical role in the pathogenesis and progression of many chronic liver diseases, such as chronic HBV and HCV infection, alcoholic liver disease, NAFLD/NASH, hepatic fibrosis, cirrhosis and liver cancer (22-24). Thirteen TLRs were identified in mammals and ten TLR members in human. Although it has reported that expression levels of TLR1-5 mRNA were significantly higher in livers of NAFLD patients in comparison to controls (25), and in fructose-induced hepatic steatosis mice, the levels of TLR1-9 increased remarkably in liver tissues (26), several studies have showed that TLR2, TLR4 and TLR9 played a role in the development of NAFLD $(12,13,27,28)$. In the
HBV transgenic mice, injections of ligands of TLR3, TLR4, TLR5, TLR7 and TLR9 inhibit HBV replication in an IFN- $\beta$ dependent manner. As we know, elevated circulating LPS and FFAs were detected in HFD-induced NAFLD models. Lipopolysaccharide was the typical and high affinity ligand to TLR4, and FFAs were also the potential ligands, which could be recognized by TLR4 and activated the downstream signal pathway.

Toll-like receptor 4 is a key component of the innate immune system and is widely expressed in all hepatic cell types, including hepatocytes, Kupffer cells, hepatic stellate cells, and sinusoidal endothelial cells. Several research studies have reported that the TLR4/MyD88 pathway is significantly associated with $\operatorname{NAFLD}(12-16,29,30)$. Myeloid differentiation factor 88 (MyD88) serves as an immediate adaptor for TLR4 in the signaling pathway, resulting in the production of TNF- $\alpha$ and IL-6, which are key regulatory molecules in the development and progression of NAFLD $(15,29-31)$.

Previous studies have shown that HBV-transgenic mice are chronic asymptomatic HBV carriers and are immunetolerant to HBV-related antigens. In this study, we have also demonstrated that an HFD, rather than HBV infection, plays a vital role in the development of NAFLD in HBV-transgenic mice $(4,21,32)$. Therefore, we hypothesized that the activation of TLR4/MyD88-mediated innate immune response may also be associated with HFD in HBV-transgenic mice. In support of this hypothesis, we established the model of HBV-transgenic mice with NAFLD and steatotic HepG2.2.15 cells to demonstrate the expressions of TLR4/MyD88 and prionflammatory cytokines. To our knowledge, this observation is significant as 
it is something that has been reported for the first time in reference to the HBV infection with NAFLD.

In our study, we observed that the activation of TLR4/ MyD88 signaling was associated with elevated expression of IL- 6 and TNF- $\alpha$ during the period 8, 16, and 24 weeks of HFD treatment. These results indicate that HFD induced hepatic inflammation through TLR4/MyD88 signaling in the HBV/NAFLD group. Recent studies uncovered that increased serum FFAs directly stimulate MyD88-dependent signaling pathway in hepatocytes $(15,16,33)$. Therefore, HFD led to elevated levels of SFAs in circulation, thereby indicating that the activation of TLR4/MyD88 signaling may also play an important role in HBV transgenic mice with NAFLD. To confirm that SFAs induced the activation of TLR4/MyD88 signaling in vitro, SA was used to induce steatosis in cell lines of hepatocytes. HepG2.2.15 cells indeed expressed TLR4, MyD88 and proinflammatory cytokines (TNF- $\alpha$ and IL-6) in response to different concentrations of SA employed in our study. These experiments provide us with the evidence that SA serves as a TLR4 ligand and consequently activates TLR4/MyD88 signaling in hepatocytes with the HBV infection.

Previous studies revealed that HBV load was negatively associated with steatosis in CHB patients with NAFLD. In our study, we also found that the viral indices (HBsAg, HBeAg and HBV-DNA) in serum samples exhibited a tendency of decrease when they were obtained from HBV transgenic mice with NAFLD; this decrease in the viral indices was especially prominent at 24 weeks. Moreover, compared with the HBV group, a significantly increased IFN- $\beta$ was detected in the serum samples obtained from the HBV/NAFLD group at 24 weeks. This indicates that the decreased viral indices may be associated with an increased IFN- $\beta$, although the level of IFN- $\beta$ has no significantly increased at 8 or 16 weeks. It has been reported that TLR4-mediated suppression of HBV replication in nonparenchymal liver cells is MyD88-independent, which can also be initiated by TLR4 activation leading to the production of IFN- $\beta$. Therefore, we speculated that LPS and FFAs, which increased in number in HBV transgenic mice with NAFLD, were sensed by TLR4 and activated through MyD88-independent pathway to form the product IFN- $\beta$ that inhibits HBV replication. The data of SA-induced steatosis in HepG2.2.15 cells were similar to that of the HBV/NAFLD group. The IFN- $\beta$ levels were elevated and the viral indices were reduced in steatoric cells, indicating that HepG2.2.15 cells were responsive to TLR4 stimulation and had the ability to produce IFN- $\beta$ through TLR4/MyD88-independent pathway in response to SFAs. In addition, the TNF- $\alpha$ and IL- 6 levels elevated in the HBV transgenic mice with experimental NAFLD and HepG2.2.15 cells with steatotic generation. Moreover, TNF- $\alpha$ and IL-6 have been reported to be the antiviral mediators $(20,34)$. Then it is indicated that IL- 6 and TNF- $\alpha$ may be another contributors inhibiting HBV replication; thus, activation of TLR4 signaling pathway may suggested to be a vital mechanism to reduce
HBV-DNA and HBV-related antigens in mice with both chronic HBV infection and NAFLD.

In conclusion, we have demonstrated that the TLR4/ MyD88 signal pathway was activated in the HBV-transgenic mice with NAFLD and HepG2.2.15 cells with SA-induced steatosis. These results further shed light on an action model in which SFA or LPS activates TLR4, in vivo and in vitro, which initiates the TLR4/MyD88 signaling and finally the production of proinflammatory cytokines. Therefore, the TLR4-mediated innate immune response may contribute to the inhibited HBV replication in experimental model with both CHB and NAFLD.

\section{Authors' Contributions}

Jian-Gao Fan and Qin Pan designed the experiments. Rui-Nan Zhang performed cytology experiments. Qin Pan and Zheng Zhang established the animal model. Hai-Xia Cao and Feng Shen tested the activity of TLR4/MyD88 signal pathway. Rui-Nan Zhang, Qin Pan, and Jian-Gao Fan drafted the manuscript. All the authors have read and approved the final revision meant for publication in scholarly journals.

\section{Funding/Support}

This work was supported by the state key development program for basic research of China (No. 2012CB517501), the Program of the Shanghai Committee of Science and Technology (No. 09140903500 and 10411956300), the 100 Talents Program of the Shanghai Board of Health (No. XBR2011007), and the National Natural Science Foundation of China (No. 81070322 and 81270491).

\section{References}

1. Wong VW, Wong GL, Chu WC, Chim AM, Ong A, Yeung DK, et al. Hepatitis B virus infection and fatty liver in the general population. J Hepatol. 2012;56(3):533-40.

2. Wong GL, Wong VW, Choi PC, Chan AW, Chim AM, Yiu KK, et al. Metabolic syndrome increases the risk of liver cirrhosis in chronic hepatitis B. Gut. 2009;58(1):111-7.

3. Rastogi A, Sakhuja P, Kumar A, Hissar S, Jain A, Gondal R, et al. Steatosis in chronic hepatitis B: prevalence and correlation with biochemical, histologic, viral, and metabolic parameters. Indian JPathol Microbiol. 2011;54(3):454-9.

4. Zhang Z, Pan Q, Duan XY, Liu Q, Mo GY, Rao GR, et al. Fatty liver reduces hepatitis $B$ virus replication in a genotype $B$ hepatitis B virus transgenic mice model. J Gastroenterol Hepatol. 2012;27(12):1858-64.

5. Fan JG, Jia JD, Li YM, Wang BY, Lu LG, Shi JP, et al. Guidelines for the diagnosis and management of nonalcoholic fatty liver disease: update 2010: (published in Chinese on Chinese Journal of Hepatology 2010;18:163-166). J Dig Dis. 2011;12(1):38-44.

6. Vuppalanchi R, Chalasani N. Nonalcoholic fatty liver disease and nonalcoholic steatohepatitis: Selected practical issues in their evaluation and management. Hepatology. 2009;49(1):306-17.

7. Machado MV, Oliveira AG, Cortez-Pinto H. Hepatic steatosis in hepatitis B virus infected patients: meta-analysis of risk factors and comparison with hepatitis C infected patients. J Gastroenterol Hepatol. 2011;26(9):1361-7.

8. Yun JW, Cho YK, Park JH, Kim HJ, Park DI, Sohn CI, et al. Hepatic steatosis and fibrosis in young men with treatment-naive chronic hepatitis B. Liver Int. 2009;29(6):878-83. 
9. Paik YH, Schwabe RF, Bataller R, Russo MP, Jobin C, Brenner DA. Toll-like receptor 4 mediates inflammatory signaling by bacterial lipopolysaccharide in human hepatic stellate cells. Hepatology. 2003;37(5):1043-55.

10. Bjorkbacka H, Kunjathoor VV, Moore KI, Koehn S, Ordija CM, Lee MA, et al. Reduced atherosclerosis in MyD88-null mice links elevated serum cholesterol levels to activation of innate immunity signaling pathways. Nat Med.2004;10(4):416-21.

11. Michelsen KS, Wong MH, Shah PK, Zhang W, Yano J, Doherty TM, et al. Lack of Toll-like receptor 4 or myeloid differentiation factor 88 reduces atherosclerosis and alters plaque phenotype in mice deficient in apolipoprotein E. Proc Natl Acad Sci U S A. 2004;101(29):10679-84.

12. Spruss A, Kanuri G, Wagnerberger S, Haub S, Bischoff SC, Bergheim I. Toll-like receptor 4 is involved in the development of fructose-induced hepatic steatosis in mice. Hepatology. 2009;50(4):1094-104.

13. Rivera CA, Adegboyega P, van Rooijen N, Tagalicud A, Allman M Wallace M. Toll-like receptor-4 signaling and Kupffer cells play pivotal roles in the pathogenesis of non-alcoholic steatohepatitis. J Hepatol. 2007;47(4):571-9.

14. Yang L, Seki E. Toll-like receptors in liver fibrosis: cellular crosstalk and mechanisms. Front Physiol. 2012;3:138.

15. Shi H, Kokoeva MV, Inouye K, Tzameli I, Yin H, Flier JS. TLR4 links innate immunity and fatty acid-induced insulin resistance. JClin Invest. 2006;116(11):3015-25.

16. Lee JY, Sohn KH, Rhee SH, Hwang D. Saturated fatty acids, but not unsaturated fatty acids, induce the expression of cyclooxygenase-2 mediated through Toll-like receptor 4. J Biol Chem. 2001;276(20):16683-9.

17. Chen Z, Cheng Y, Xu Y, Liao J, Zhang X, Hu Y, et al. Expression profiles and function of Toll-like receptors 2 and 4 in peripheral blood mononuclear cells of chronic hepatitis B patients. Clin Immunol. 2008;128(3):400-8.

18. Isogawa M, Robek MD, Furuichi Y, Chisari FV. Toll-like receptor signaling inhibits hepatitis B virus replication in vivo. J Virol. 2005;79(11):7269-72.

19. Wu J, Lu M, Meng Z, Trippler M, Broering R, Szczeponek A, et al Toll-like receptor-mediated control of HBV replication by nonparenchymal liver cells in mice. Hepatology. 2007;46(6):1769-78.

20. Sweet MJ, Hume DA. Endotoxin signal transduction in macrophages. J Leukoc Biol. 1996;60(1):8-26.

21. Zheng FJ, Fu YH, Liu GZ, Zhou JH, Kong XP. Study on the immune tolerance status and blood biochemical index of hepatitis B vi- rus transgenic mice. Chin J Infect Dis. 2011;29:641-7.

22. Schwabe RF, Seki E, Brenner DA. Toll-like receptor signaling in the liver. Gastroenterology. 2006;130(6):1886-900.

23. Seki E, Brenner DA. Toll-like receptors and adaptor molecules in liver disease: update. Hepatology. 2008;48(1):322-35.

24. Broering R, Lu M, Schlaak JF. Role of Toll-like receptors in liver health and disease. Clin Sci (Lond). 2011;121(10):415-26

25. Kanuri G, Ladurner R, Skibovskaya J, Spruss A, Konigsrainer A Bischoff SC, et al. Expression of toll-like receptors 1-5 but not TLR 6-10 is elevated in livers of patients with non-alcoholic fatty liver disease. Liver Int. 2015;35(2):562-8.

26. Wagnerberger S, Spruss A, Kanuri G, Volynets V, Stahl C, Bischoff SC, et al. Toll-like receptors 1-9 are elevated in livers with fructoseinduced hepatic steatosis. BrJ Nutr. 2012;107(12):1727-38.

27. Szabo G, Velayudham A, Romics LJ, Mandrekar P. Modulation of non-alcoholic steatohepatitis by pattern recognition receptors in mice: the role of toll-like receptors 2 and 4. Alcohol Clin Exp Res. 2005;29(11 Suppl):140S-5S

28. Miura K, Kodama Y, Inokuchi S, Schnabl B, Aoyama T, Ohnishi $\mathrm{H}$, et al. Toll-like receptor 9 promotes steatohepatitis by induction of interleukin-1beta in mice. Gastroenterology. 2010; 139(1):323-34 e7.

29. Miura K, Seki E, Ohnishi H, Brenner DA. Role of toll-like receptors and their downstream molecules in the development of nonalcoholic Fatty liver disease. Gastroenterol Res Pract. 2010;2010:362847.

30. Song CY, Zeng X, Wang Y, Shi J, Qian H, Zhang Y, et al. Sophocarpine attenuates toll-like receptor 4 in steatotic hepatocytes to suppress pro-inflammatory cytokines synthesis. J Gastroenterol Hepatol. 2015;30(2):405-12.

31. Roh YS, Seki E. Toll-like receptors in alcoholic liver disease, nonalcoholic steatohepatitis and carcinogenesis. J Gastroenterol Hepatol. 2013;28 Suppl 1:38-42.

32. Guidotti LG, Matzke B, Schaller H, Chisari FV. High-level hepatitis B virus replication in transgenic mice. J Virol. 1995; 69(10):6158-69.

33. Li L, Chen L, Hu L, Liu Y, Sun HY, Tang J, et al. Nuclear factor highmobility group box1 mediating the activation of Toll-like receptor 4 signaling in hepatocytes in the early stage of nonalcoholic fatty liver disease in mice. Hepatology. 2011;54(5):1620-30.

34. Hosel M, Quasdorff M, Wiegmann K, Webb D, Zedler U, Broxtermann $\mathrm{M}$, et al. Not interferon, but interleukin-6 controls early gene expression in hepatitis B virus infection. Hepatology. 2009;50(6):1773-82. 